\title{
Daily cost of delay to adequate antibiotic treatment among patients surviving a hospitalization with community-onset Acinetobacter baumannii pneumonia or sepsis
}

\author{
Marya D. Zilberberg ${ }^{1 *}$, Brian H. Nathanson², Kate Sulham³ ${ }^{3}$ Weihong Fan ${ }^{3}$ and Andrew F. Shorr ${ }^{4}$ \\ See related research by Zilberberg et al. https://ccforum.biomedcentral.com/articles/10.1186/s13054-016-1392-4
}

With $>80 \%$ prevalence of multi-drug resistance, Acinetobacter baumannii (AB) poses a serious public health threat $[1,2]$. We recently showed that inappropriate empiric therapy in the setting of community-onset $A B$ pneumonia or sepsis is associated with $80 \%$ increase in hospital mortality [3]. The economic effects of delay in appropriate treatment are less clear. In a subgroup of the same cohort, we explored the cost associated with each day's delay after obtaining index culture in instituting adequate therapy.

The original cohort derived from 176 US hospitals in the Premier Research database 2009-2013 and consisted of all adult patients admitted with pneumonia or sepsis as principal diagnosis, or as a secondary diagnosis in the setting of respiratory failure, along with antibiotic administration within 2 days of admission [3]. Only culture-confirmed infections were included. Inappropriate empiric therapy was present if the antibiotic administered did not cover the organism or if coverage did not start within 2 days of obtaining the positive culture. For the current analysis, patients were excluded if they did not survive the hospitalization or never in the hospitalization received adequate treatment (an agent that covered $\mathrm{AB}$ ). "Day 0" to adequate therapy was the day the positive culture was obtained. To assign costs to delay in adequate treatment, we categorized length of stay (LOS) into three groups, as number of days: (1) until the first index culture ("pre" time); (2) after the index culture until the first appropriate antibiotic (period

* Correspondence: evimedgroup@gmail.com

${ }^{1}$ EviMed Research Group, LLC, PO Box 303, Goshen, MA 01032, USA Full list of author information is available at the end of the article of interest); and (3) after the first appropriate antibiotic until hospital discharge ("post" time). We adjusted for pre and post times so that the costs associated with them were not attributed to the period of interest. The model structure was a generalized linear model (GLM) with a logarithmic link to account for the skew in total costs. In addition to the time variables, as in our prior study, covariates included other parameters known by hospital day 2 [3].

Of the 1423 patients in the original cohort, 460 $(32.3 \%)$ were included in the current analysis. Among these, only $201(43.7 \%)$ received appropriate therapy on day 0 , with the median time to adequate treatment 3 days (interquartile range 1,5 ). In the GLM, each day's delay in instituting adequate therapy added $\$ 1344$ (95\% confidence interval $\$ 423, \$ 2266, p=0.004)$ to the total cost of hospitalization.

This analysis illustrates that delaying appropriate empiric treatment carries a financial cost that begins to accrue the moment infection is suspected and culture obtained. The fact that fully one-half of our population took $\geq 3$ days to receive appropriate treatment, totaling $>\$ 4000 /$ patient, equates to a substantial expenditure. Currently, in order to improve the likelihood of appropriate empiric coverage, and, in turn, the chances of survival, the only viable choice is to administer broad-spectrum therapy. However, since newer antibiotics with broader spectra are necessarily more expensive than older generic options, there is hesitation associated with the employment of newer alternatives. Our findings may refute the proposition that withholding novel agents represents a costminimization tactic. 


\section{Abbreviations}

AB: Acinetobacter baumannii; LOS: Length of stay

\section{Acknowledgements}

No one other than the listed authors participated in the study design, analysis, interpretation, or manuscript drafting or revision.

\section{Funding}

This study was supported by a grant from The Medicines Company, Parsippany, NJ, USA.

Availability of data and materials

Not applicable.

\section{Authors' contributions}

MDZ contributed substantially to the study design, data interpretation, and the writing of the manuscript. BHN contributed substantially to the study design, data interpretation, and the writing of the manuscript. He had full access to all of the data in the study and takes responsibility for the integrity of the data and the accuracy of the data analysis. KS contributed substantially to the study design, data interpretation, and the writing of the manuscript. WF contributed substantially to the study design, data interpretation, and the writing of the manuscript. AFS contributed substantially to the study design, data interpretation, and the writing of the manuscript. All authors read and approved the manuscript

\section{Competing interests}

MDZ is an employee of EviMed Research Group, LLC, and has served as a consultant to and/or received research funding from The Medicines Company, Pfizer, Astellas, Tetraphase, Theravance, Merck, Shionogi, Archeogen. BHN is an employee of OptiStatim, which has received funding from EviMed to conduct this study. KS is an employee and stockholder of The Medicines Company. WF is an employee and stockholder of The Medicines Company. AFS has served as a consultant to, received research support from, or been a speaker for Abbott, Actavis, Alios, Astellas, AstraZeneca, Bayer, BMS, Cardeas, Medicines Company, Merck, Pfizer, Roche, Tetraphase, Theravance, and Wockhardt Pharma, Shionogi.

\section{Consent for publication}

Not applicable.

Ethics approval and consent to participate

Because this study used already existing HIPAA-compliant fully de-identified data, it was exempt from IRB review.

\section{Sponsor role}

Although KS and WF are employees of the sponsor and participated in the study as co-investigators, the larger sponsor had no role in study design, data analysis, or interpretation or publication decisions.

\section{Publisher's Note}

Springer Nature remains neutral with regard to jurisdictional claims in published maps and institutional affiliations.

\section{Author details}

'EviMed Research Group, LLC, PO Box 303, Goshen, MA 01032, USA. ${ }^{2}$ OptiStatim, LLC, Longmeadow, MA, USA. ${ }^{3}$ The Medicines Company, Parsippany, NJ, USA. ${ }^{4}$ Washington Hospital Center, 110 Irving St NW, Washington, DC 20010, USA.

\section{Published online: 05 June 2017}

\section{References}

1. CDDEP: The Center for Disease Dynamics, Economics and Policy. Resistance Map: Acinetobacter baumannii Overview. http://www.cddep.org/projects/ resistance_map/acinetobacter_baumannii_overview. Accessed 8 Jan 2016.

2. Zilberberg MD, Kollef MH, Shorr AF. Secular trends in Acinetobacter baumannii resistance in respiratory and blood stream specimens in the United States, 2003 to 2012: a survey study. J Hosp Med. 2016;11:21-6.

3. Zilberberg MD, Nathanson BH, Sulham K, Fan W, Shorr AF. Multidrug resistance, inappropriate empiric therapy, and hospital mortality in Acinetobacter baumannii pneumonia and sepsis. Crit Care. 2016;20:221. 